\title{
Towards a model of Collaborative Support for Distance Learners to Perform Joint Tasks
}

\author{
B.Barros, M.Rodriguez-Artacho and F.Verdejo \\ UNED - Dep de Ingeniería Eléctrica, Electrónica y Control \\ Ciudad Universitaria $s / n 28040$ Madrid Spain \\ e-mail: bbarros, martacho,felisa \{@ieec.uned.es\}
}

\begin{abstract}
This paper describes the design and implementation of a prototype to support collaborative learning. It is part of a larger effort dealing with the study of mechanisms of cooperation and collaboration between distance learners while performing joint tasks involving complex interactions. The final goal of the project is to produce a conceptual model and a tailorable system for describing a variety of collaborative distance learning scenarios providing active group support. A first web-based prototype integrating private and shared workspaces has been implemented, and a testbed carried out with graduate students. The learning scenario and the collaborative facilities offered by the system are presented.
\end{abstract}

\section{Keywords}

Computer supported collaborative learning, Distance learning, Cooperative tools, Higher education, Coordination 


\section{INTRODUCTION}

The shift from individual to collaborative learning is an emerging approach in recent learning research and experiments. Distance learning is one setting in particular that deserves more and more attention due to the spread of web-based facilities. The scenario may include: a) a group of students performing different kinds of cooperative learning activities such as discussion or problem-solving, using conventional or multimedia-based material; b) a group of teachers, organising, facilitating and supervising the cooperative learning; c) a group of authors creating courseware and sources of information; and d) a telecomputer environment supporting all these activities.

Collaborative learning is based on the idea that learning occurs when students have to explain, develop or justify their ideas to attain a shared goal. The variation on the underlying model of learning is from a classical information-processing and transmission oriented model to a more social-process orientation centered on promoting learning through constructive activities (doing) and interactions (communicating) in a meaningful context.

Distance collaborative learning can be shaped in different ways (Verdejo, Cerri 1994), a joint project is appealing for a range of scientific and engineering subjects. Proposals for computational group support differ in the type of structure and the degree of genericity they offer to users, ranging from general purpose tools for communication and shareworking to fully typified spaces with specific primitives and communication protocols related to the task at hand. To mention just a few of the current educational projects exploring this area, Edelson et al. (Edelson et al, 1996) offer a shared Notebook with a set of page types corresponding to a task model of scientific inquiry to be used for collaborative science learning. The page types include questions, conjectures, evidence for and against, information, commentary as wells as plans and their steps. Students can create instances of these pages and link them as their work progresses. A learner interface built upon their scientific inquiry model, suggest the next steps for a student to pursue. Wan et al. (Wan, Johnson 1994) report on CLARE, an environment supporting the task of learning to understand scientific literature. They define an explicit process model: summary, evaluation, comparison, argument and integration and provide specific representations and structure for each of these individual and group activities.

Our aim is to explore collaboration at a distance for a range of tasks involving complex interactions, and to study in which ways an active computer-based support could benefit the learning process. Although we do not exclude interaction in real time, we focus on asynchronous interactions because this is the preferred mode for real distance learning students. They usually have tight time constraints making it difficult to establish common real time slots for groupworking. 


\section{THE PROJECT AIM AND BACKGROUND}

This paper describes the design and experimentation of a prototype to support collaborative learning. It is part of a larger effort ${ }^{1}$ dealing with the study of mechanisms of cooperation and collaboration between distance learners while performing a joint task. The final goal of the project is to produce a conceptual model for describing a variety of collaborative distance learning scenarios based on active support. A tailorable system built on this model, will provide functionality to generate an adapted computational environment to perform personal and shared activities for specific learning purposes. The project methodology includes iterative steps of prototype design, user experimentation and evaluation. Our approach benefits from a diversity of sources, mainly linked to three research streams (1) learning as a process of social knowledge construction (Scardamalia, Bereiter 1993), where students could work cooperatively in a context and with problems relevant to real practice (Schank, Kass 1996) (2) activity and conversational models from $\mathrm{CSCW}$, proposed and implemented for teamwork in professional environments (De Cindio et al 1988) (Winograd, Flores 1986) (Ellis et al 1991) (Manole, Fry 1992) (Collis, 1994) and (3) distributed cognition theories (Coelho 1996) to model expertise and behaviour of a group of interacting autonomous agents to execute a task.

In this paper we will describe a first prototype and a preliminary testbed set up in our University. The next section defines the type of learning situations we have considered. In section four, the main features of the current prototype are outlined. We will conclude with a summary of current and planned experimental work.

\section{THE LEARNING SITUATION}

We will characterize the situation as a set of small ( 2 to 4$)$ groups of graduate students, geographically distributed, carrying out together a learning task in a period of time. A computer environment providing a variety of resources and tools, some of them shared, offers to each group of students support to carry out the learning activities in collaboration. Teachers are also available though their intervention is primarily to give response to explicit learner's requests and to detect deadlocks in groups. At the moment their main role is to prepare the learning tasks and to observe and analyze the whole process, in order to give feedback to designers for further development and experimentation.

We distinguish two learning tasks, one content-oriented, related to a knowledge domain, the other one related to the ability to work cooperatively, including skills such as being able to propose, explain and discuss ideas with co-workers through

\footnotetext{
'The project includes a Spanish-Portuguese collaboration. Participants in the project are : B.Barros, A. Garcia Serrano, M. Rodriguez, and F. Verdejo from Spain. H. Coelho and A. Paiva from Portugal. Users are graduate and Ph.D students belonging to two Portuguese Universities and two Spanish Universities.
} 
electronic tools, to negotiate and manage a plan to achieve a task, to establish and follow a common schedule to reach a goal in due time.

The learning situation involves personal and joint work; for the first experiment, students have to read and understand a collection of papers and elaborate a synthesis of the main ideas. It is recommended to students that they should organize their work into two main phases, a first phase centred on reading and understanding the material individually, a second one to elaborate a paper in collaboration with their peers. In further experiments a third phase would be added: comparing and evaluating their results with others' group work.

For the first phase, defined mainly as personal work, communication is also encouraged. Questions, doubts, clarification, further information can be interchanged within the student group to facilitate to each other a better understanding of the material.

In the second phase students have to use their understanding to elaborate a synthesis of the papers. Their final product, a document in this case, has to be written according to the topic contents and structure specified by the teacher. They can create the essay in an incremental way, section by section. For each section they can open a period of discussion, interchanging and refining proposals, and once they arrive at an agreement about a written version for that section, the text is automatically integrated into the document.

The prototype offers to each student a private workspace and an agenda and, to each group two interlinked but separated shared spaces: the working space and the communication space. Each space provides different functions.

The main events of the learners' interactions are recorded by the system. In this way tutors can analyze not only the final results obtained by the students but also the processes carried out by the group. For the experimentation phase this information is crucial, a main goal of the project is to determine, describe and evaluate how the cooperation works and in which way it can be facilitated or improved. What to gather and the level of abstraction required representing events and their links in order to perform learning task analysis is still an open matter. Too much fine grained information can cause an overflow of data, difficult to handle without automatic filtering techniques. At this point we have decided to include in the prototype three types of facilities:

(1) The explicit notification (performed automatically by the system on each personal agenda) of new contributions happening within each group. This warning is not only useful for learners, to be aware of the activity going on within its group, but also helps teachers to have a quick overview of the task evolution

A shared summary of the group activity to accomplish their work in form of:

(2) an automatically structured index of the set of contributions, labelled with author' s names 
(3) an automatically updated index of the semi-structured set of messages interchanged by the members of the group while performing the learning task.

\section{THE SYSTEM PROTOTYPE}

We have implemented a first web-based prototype to support distance learning students to elaborate a synthesis paper through a structured coedition process. The system supports two kind of users/roles: students to perform joint activities related to a learning task and teachers to prepare the task and to follow and analyze the students' activities and results. In this paper we will present the prototype services for the students.

The prototype has been designed taking into account four claims (1) collaboration takes the form of a structured discussion between peers, without explicit moderator role. A set of conversational primitives allows the group to organize their joint work process. However (2) coordination between students (and teachers) should be done using flexible but explicit mechanisms to clearly state responsibilities and commitments. (3) The task for each student group is to produce a common document sharing a common workspace. (4) All the information generated in the process should be accessible, extractable and visualized from a variety of viewpoints, such as group related, time related, student related, task related.

Our aim is to offer a system supporting the exchange of information, helping to co-ordinate the discussion and letting the group work at its ownpace, to generate a common essay. To achieve these objectives, we have two separate but interlinked shared spaces: the coedition space and the coordination space. Nevertheless the prototype offers also in an integrated way a private, individual workspace, to each student.

\subsection{Individual Workspaces}

Two options of the bar menu provide access to private areas: "individual work" offers to users a kind of personal notebook while "agenda" allows the representation and management of information about activities and commitments.

Figure 1 shows the screen when the option individual work has been selected by one of the students participating in the testbed. 


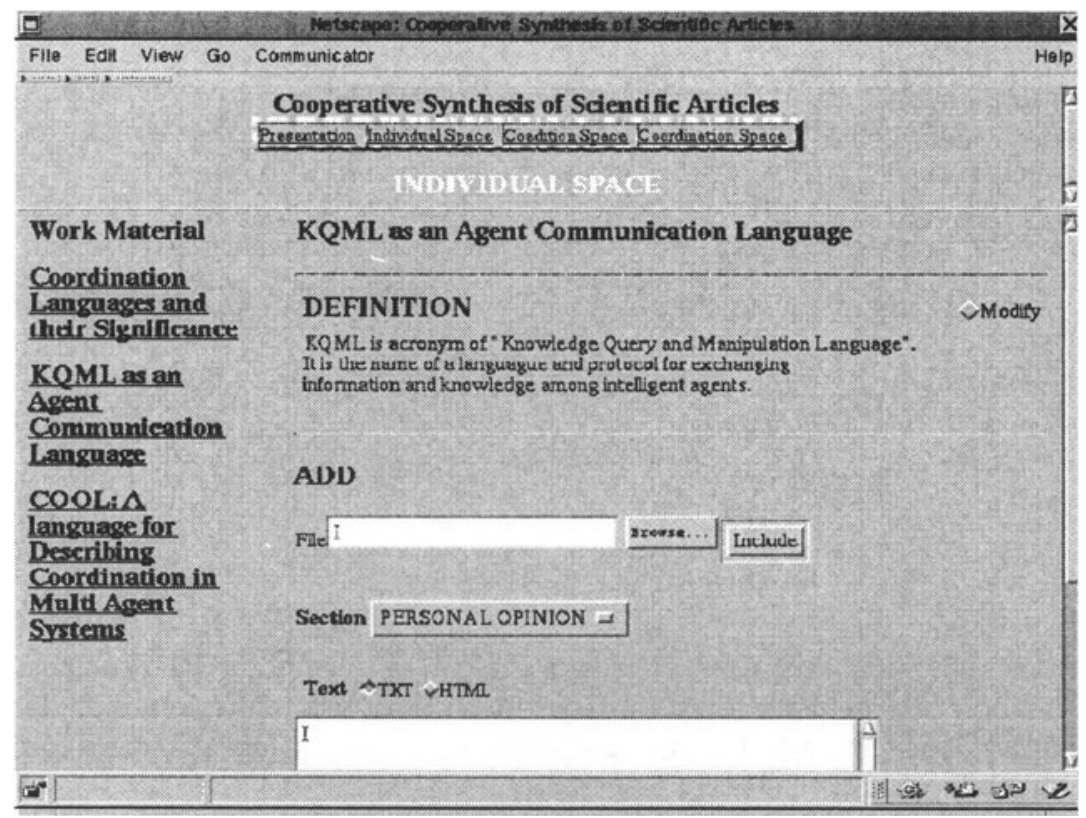

Figure 1 Individual Workspace

On the left part appears the list of papers assigned to its group. During the personal phase, he has to read them and in this case to write for each paper a brief summary, following some key points provided by the teacher. The list of titles works as an index. Clicking on one of the titles the pages of the notebook corresponding to the paper summary become available on the upper right part of the screen. The pages are empty at the beginning of the task and can be created by the student in an incremental way using a standard set of editing operations. Pages of the notebook can either be forms (predefined by the teacher) or have free format.

\subsection{Shared Workspaces}

Collaboration is required for carrying out a joint task, the prototype provides two options of the bar menu for such purpose: collaborative synthesis and coordination. Both include shared spaces belonging to each working group, we call them working space and coordination space respectively. The last one includes a Bulletin Board service, allowing the posting of messages for the group.

The working space consists of three related subspaces: a coedition space for the draft version been currently discussed by the group, a final version space for storing portions of the synthesis document considered as finished parts by common agreement, and a history space to store other previous draft versions, although discarded at some time, they are nevertheless available for an eventual reconsideration. 


\subsubsection{Workspaces components}

The production of an essay can follow a variety of approaches, ranging from a well established plan of work to a completely unstructured process. An example of the former could be to organize the task in form of subtasks like: global brain storming to fix key ideas, writing a schema, develop the content for each section, revision and refinement of the text, etc. When this same task has to be done collaboratively, the process become more complex because not only the approach and the task management have to be agreed and successfully accomplished by the members of the group, but also each substask can be matter of argument and negotiation.

Let us assume that the learning task starts from a given schema, provided by the teacher in this case, and students should focus on the writing of the content of each section. We can consider this phase as a coediting task, where the students should participate in writing their own proposals, discussing the peer proposals, commenting, mutually asking questions and giving answers until this process converges to a written text satisfactory to the group.

A way of providing some structure to organize automatically the contributions and arguments generated during the writing discussion is to type each student contribution, so as to link and thread a set of related interchanges in the Coedition Space. We propose coedition as a structured discussion process where group members interact with each other to create a content section by means of the following types of contributions:

- Proposal: the first contribution

- Contraproposal: alternative text for an existing proposal. It is another original proposal or made by extending an existing proposal

- Question: someone asks for something related to an annotation.

- Comment: text for commenting an existing annotation.

- Clarification: text for answering a question or for extending the information on an existing annotation

- Agreement: annotation where the user declares his agreement with another annotation

\subsubsection{The structure of the co-edition space}

As stated before the Coedition process has two phases for every section, namely construction and agreement. During the former, the group members propose ideas or comments, they write, refine and polish a text. The first annotation becomes a proposal. This proposal can be made by any member of the group. Once a proposal is there, students can make contributions, contraproposals, questions, comment or clarifications. New contributions can be linked to these, and so, a tree named coedition process scheme is automatically generated. This scheme is a representation of the group dynamic.

In figure 2, we can see a snapshot of the Coedition Space during the discussion phase. 


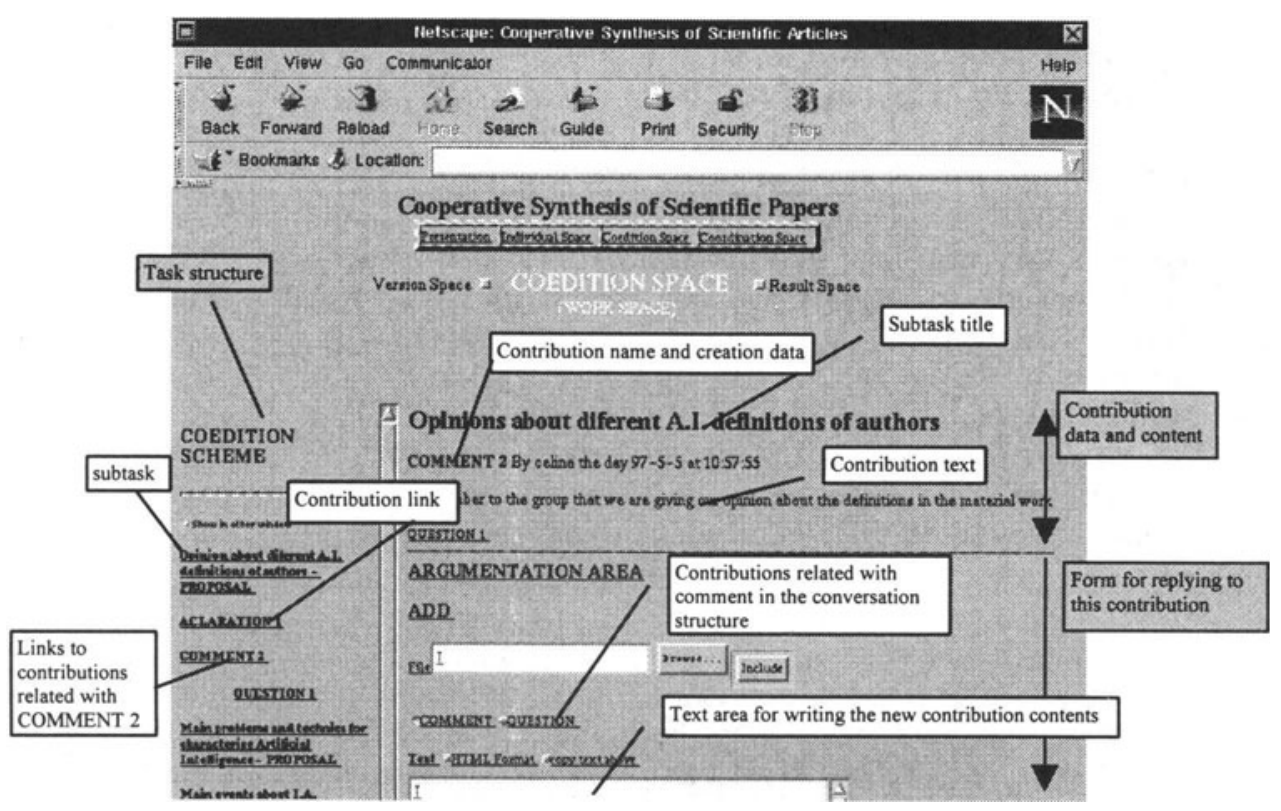

Figure 2 Coedition Space

On the left part the coedition process scheme is shown. It contains part of the content, and on the right part, - after clicking on one of the contribution names - the whole content for this contribution. At the top of the right part there is the annotation information, below the names of contributions related to it, and below, a form where the student can write a new contribution if he wishes.

While working on the coedition process, an image of the current state of the Coedition Space can be saved in the Version Space. Figure 3, shows the Version Space interface appearing when this option is clicked on the bar menu.

On the left, the available operations in this workspace and the list of existing versions appear. The user can see a complete old version contents by clicking on its name.

The following Version Space operations allow for the interchange of information between both spaces:

- STORE: primitive that stores the current state of the Coedition Space as a new version in the Version Space. The result is a new version

- BACK primitives for restoring a version in Version Space to the Coedition Space 


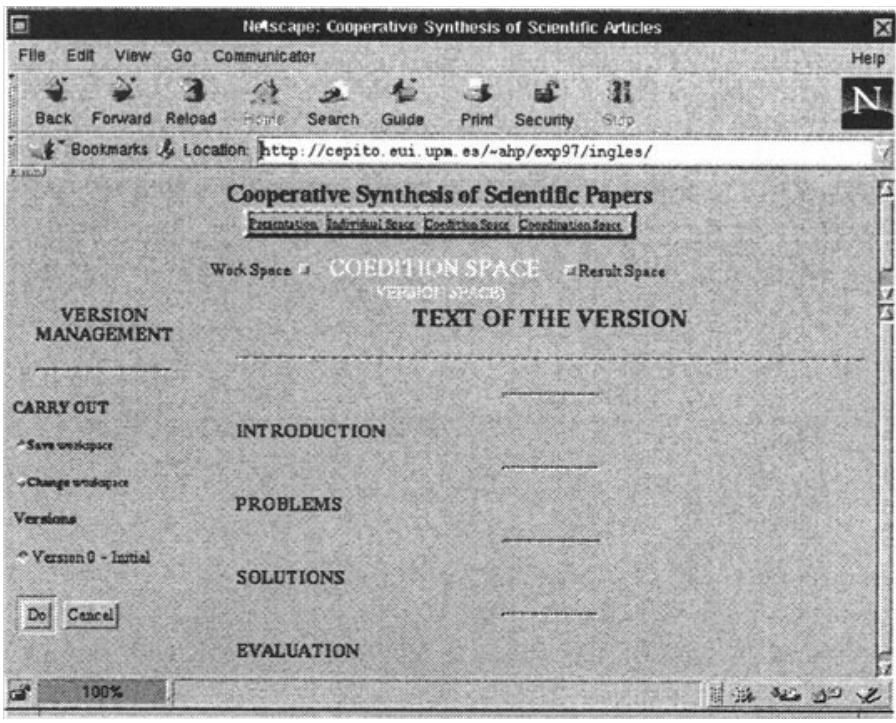

Figure 3 Version Space

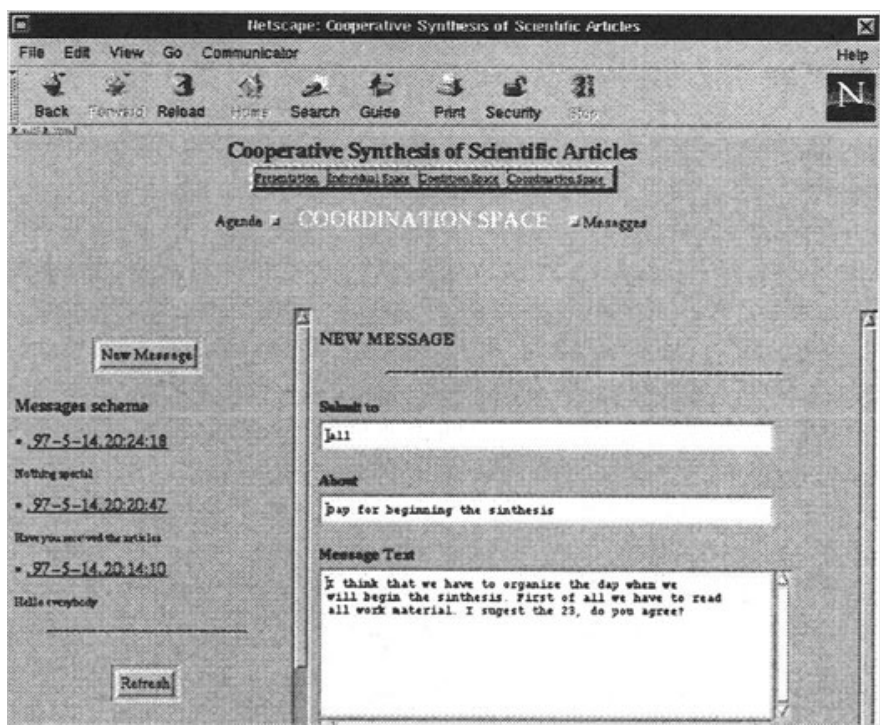

Figure 4 Notice Board Space 


\subsubsection{The Coordination Space}

The coordination space includes a Notice Board Space and an agenda.

- The Notice-Board Space

Here the group members can post messages about their plans, comments about the work, as well as general management affairs. Figure 4 shows the Notice-Board Space. On the left, there is a list of messages ordered by data. If the user clicks on one message title, the whole message contents will be displayed on the right side: title, content, author, and sending data.

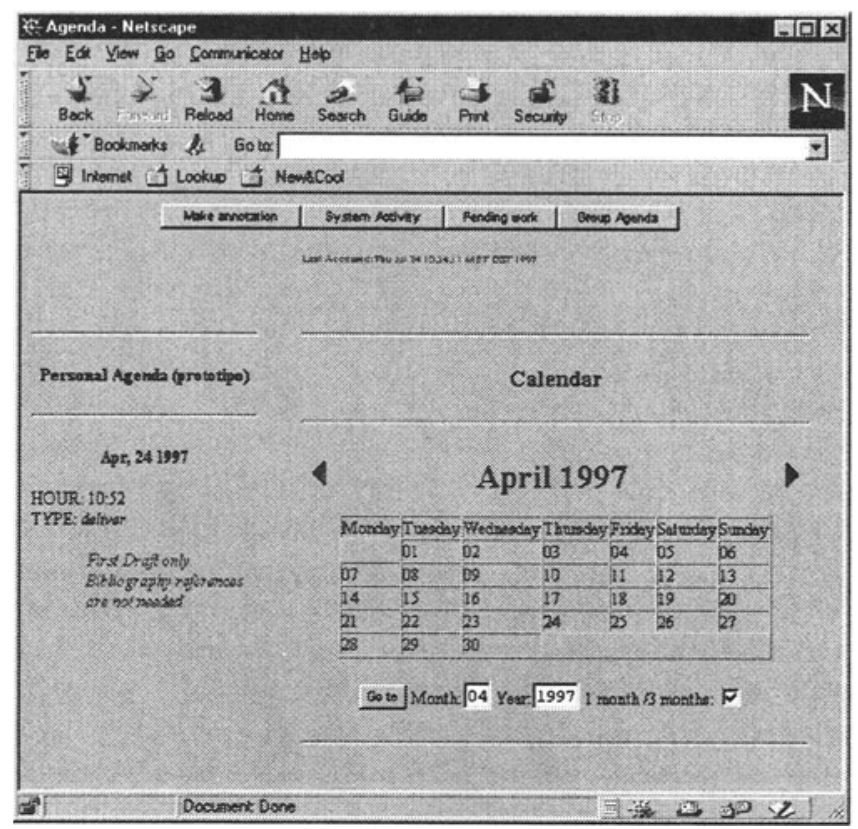

Figure 5 Student Agenda

\section{- Agenda}

The Agenda is a tool integrated within the Coordination Workspace. As mentioned in previous sections, the agenda organizes and updates information relative to the development of the joint task.

During this process of development, the user has to plan his individual and group activities, annotate some information, arrange deadlines with others members of the group, etc. Additionally, when a user logs onto the system, it is useful to know what have been the last activities performed by the other members of the group and which are the new contributions to the task at hand. 
Thus, it becomes necessary to offer a way to plan both individual and group work by means of a tool allowing the user to (a) schedule the activities involved in performing a given task, whether they are part of a personal scheduling, or cooperative activities, and (b) trace the development of the task and the activity of the members of the group in order not only to inform students but also to facilitate the subsequent analysis of the process by the teacher.

Figure 5 shows a snapshot of a student agenda, obtained by clicking the option agenda in the coordination space window. The upper part contains a menu bar, with four options: make annotation, system activity, pending mark and group agenda. The bottom right part visualizes the calendar by months. Clicking on a day, the appointments of the personal agenda for the selected day are shown on the left side.

Both personal and group annotations in the agenda are typed in order to classify and distinguish the different kind of information that a user may need to manage for scheduling his personal and group work. Selecting the make annotation option of the menu bar opens a window allowing the creation of a personal annotation as shown in figure 6.

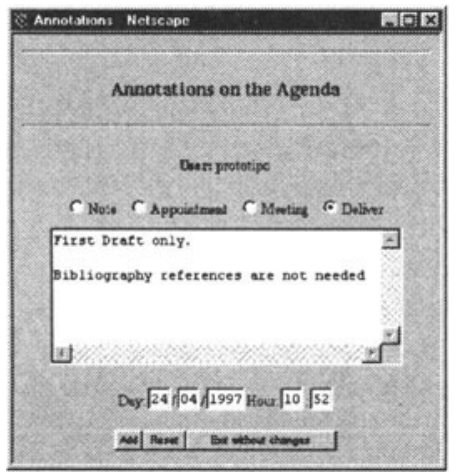

Figure 6. Window for creating a new annotation in the agenda

The prototype considers four types of agenda annotations :

- NOTE : Information to remember. Acts as a "post-it" note.

- APPOINTMENT: Two or more members decide that the group or some of its members have to perform a synchronous activity

- MEETING: There is a synchronous meeting to discuss the development of the task

- DELIVER: There is deadline to deliver a given work.

The agenda is updated in two manners, by the user whenever he makes annotations in his personal agenda, and by the system, that, in addition, makes annotations automatically inherited from the group agenda. 
The types of contributions can be enriched according to the nature of the joint task. The choice of these four types is however a first step in distinguishing different types of information that a user may need to annotate, but at the same time, this set can be modified as the analysis of the system activity reflects that a more or less extensive set of types is needed. Furthermore, it becomes important to notice that very structured and enriched information involves also more complexity to the interface and this can lead to laziness in the use of the system as the user can get tired of clicking and popping too many buttons or menus.

As mentioned before, the system also offers to the user a list of the new contributions made by the other partners since his last login (system activity option on the bar menu in figure 5). This additional feature of the agenda is useful for the student as it allows him to know the progress of the joint task, and also for the supervisor as it constitutes interesting information about the behaviour of the group members.

In short, the agenda offers a high perspective of the performed task, much better than a simple scheduler of the user work. This constitutes a first step in the organization of a structured scheduling of the development of a joint task. Much more work has to be done in this aspect in order to anticipate some behaviour patterns allowing the system to offer active support to dynamize the group.

\section{FUTURE WORK}

First sets of experiences have been organized in April-May 97. Three groups of PhD students have been working together to prepare an essay on A.I. topics. Each group (two to three students located in different towns) used the system for three weeks. The aim of this first pre-test was to check the accessibility and reliability of the prototype, as well as the acceptability from the students. This pre-test has been successful and next term a wider set of experiences will be carried out. The experiences will involve PhD students and tutors in a similar scenario but in three different universities. We expect more diversity in this trial, both from students and tutors. After this second technical pre-test the experimental phase will consist of a series of evaluation experiences, focusing on the analysis of the collaboration process. The results of the educational investigation will be discussed and their interpretation will help to enhance the design of the final version of the software. Our plan is to have the system operative for general use at the UNED server.

\section{ACKNOWLEDGEMENTS}

This work has been partially funded by the Ministerio de Educación y Cultura through Acción Integrada HP97-0050 and by CICYT (Comisión Interministerial de Ciencia y Tecnología), Spain, project number TEL97-0328-C02-01. 


\section{REFERENCES}

Coelho, H. (1996) Distributed coginition. Panel on Proceedings of EUROAIED.

Collis B. (1994) Collaborative learning and CSCW: research perspectives for interworked educational environments, in Lessons from learning. (ed. R.Lewis and P.Mendelsohsn), North-Holland. 1994.

De Cindio, F., Simone C. Vassallo and R. Zanaboni, A. (1998) CHAOS: a knowledge based system for conversing inside office, in IFIP tc8/WG 8.4 Int Workshop on Office Knowledge: representation, management and utilization. (ed. W. lamersdorf), North-Holland 1988.

Edelson,D.C., Pea R.D. and Gomez L.M. (1996) The collaboratory Notebook. Communications of the $A C M, 39$, No 4, 32-33.

Ellis, C.A., Gibbs, S.J.and Rein, G.L. (1991) Groupware: some issues and experiences. Communications of the ACM, 34, No. 1, 38-58.

Malone, T.W. and Fry,C. (1992) Experiments with OVAL: a radically tailorable tool for cooperative work, in $C S C W 92$ Proceedings. (ed. M.Mantei, R.Baeker), 289-297.

Scardamalia M., Bereiter C. (1993) Technologies for knowledge-Building discourse. Communications $A C M, 36$, No. 5, 37-41 .

Schank R.C. and Kass, A.(1996) A goal-based Scenario for High School Students. Communications of the ACM, 39, No 4, 28-29.

Verdejo, M.F. (1996) Interaction and collaboration in Distance Learning through computer mediated technologies. In Advanced Educational Technology: Research Issues and Future Potential. (ed. T.Liao ). Computer and System Sciences, 145, Springer-Verlag, 77-88.

Winograd, T. (1986) Flores F. Understanding computers and cognition: a new foundation for design. Norwood Ablex.

Wan D. and Johnson P. (1994) Experiences with CLARE: a computer-supported collaborative learning environment. Int. J. Human-Computer Studies, 41, No. $6,851-879$.

\section{BIOGRAPHIES}

Beatriz Barros received her Computer Science degree in 1994 at Technical University of Madrid, and now she is working for her Ph.D. in Artificial Intelligence. She is Assistant Professor at UNED since 1996. Her research interest areas are computer supported cooperative systems, collaborative learning and distance education.

Miguel Rodríguez-Artacho is Assistant Professor at the Universidad Nacional de Educación a Distancia. He received his Computer Science Engineer Degree from the Technical University of Madrid in 1994.Now he is working in his Master of Science Degree on Telecommunication and Network Systems and also in his Ph. D. about architectures for distance learning environments. 
M.Felisa Verdejo is Professor of Computer Science at the Universidad Nacional de Educacion a Distancia, UNED, since 1991. She received a 3rd-cycle Thesis in Computing Science from the University Pierre et Marie Curie (Paris, Francia) in 1976, and a Ph.D in Sciences at the Universidad Complutense (Madrid, Spain) in 1981. She was formely professor at the Universities of El Pais Vasco (San Sebastian, Spain) and Politécnica de Cataluña (Barcelona, Spain). Her main research interests are in the areas of Natural Language Processing, and Interactive Systems to support human learning. Major national and international projects she has been involved related to the last topic are: CAPRA an intelligent tutoring system for novice programming, EPOS, developing european learning through technological advance, TOSKA, tools and methods for knowledge based authoring ESCAD, a computer environment to support cooperative work for distance learning activities, and STEED, telematics support for learning at a distance. She is co-editor of the book Collaborative dialogue technologies in distance learning. (Vol 133, Nato ASI Series, Springer-Verlag 1994) and has published several papers on these subjects. 